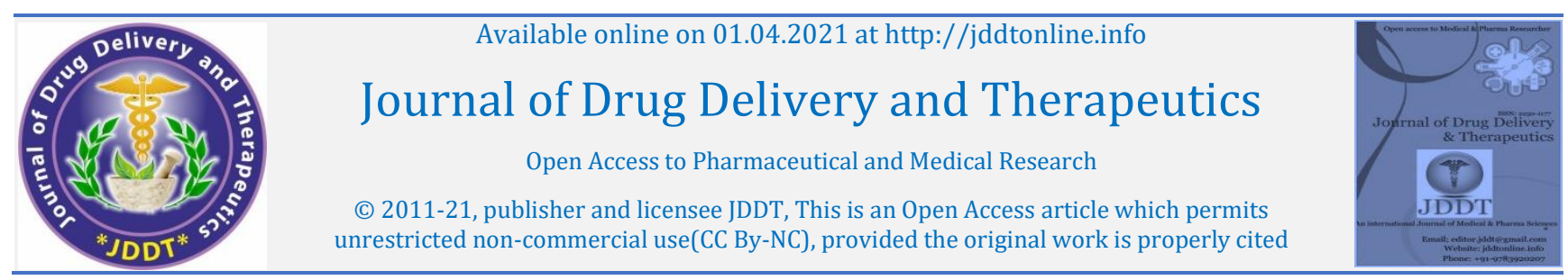

(C) 2011-21, publisher and licensee JDDT, This is an Open Access article which permits
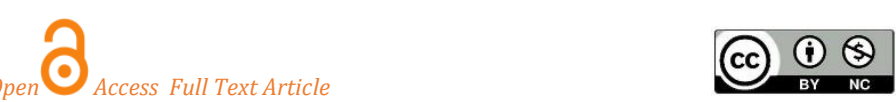

Short Communication

\title{
HCIP: An Online database for prediction CYP450 Enzyme Inhibition potential of bioactive compounds
}

Sarvesh Sabarathinam*, Preethi L, Haripritha Meganathan, Arjun Gokulan M, Dhivya Dhanasekaran, Nila Ganamurali, Rahul Radhakrishnan SRM College of Pharmacy, SRM Institute of Science and Technology, Kattankulathur-603 203, Tamil Nadu, India

\section{Article Info:}

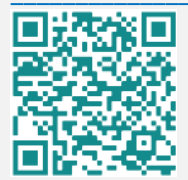

Article History:

Received 13 Feb 2021;

Review Completed 21 March 2021

Accepted 26 March 2021;

Available online 01 April 2021

Cite this article as:

Sabarathinam S, L P, Meganathan H, Gokulan M A, Dhanasekaran D, Ganamurali N, Radhakrishnan R, HCIP: An Online database for prediction CYP450 Enzyme Inhibition potential of bioactive compounds, Journal of Drug Delivery and Therapeutics. 2021; 11(2):253-255 DOI: http://dx.doi.org/10.22270/jddt.v11i2.4637

\section{*Address for Correspondence}

Sarvesh Sabarathinam, M.Pharm., (PhD)Research scholar, Department of Pharmacy Practice, SRM College of Pharmacy, SRM Institute of Science and Technology, SRM Nagar, Kattankulathur-603 203, Kancheepuram,

Tamil Nadu, India

\section{Abstract}

Background: Concomitant administration of herbal medicine and conventional may lead to severe metabolism-oriented herb-drug interactions. However, detecting herb-drug interaction is expensive and higher time-consuming. Several computer-aided techniques have been proposed in recent years to predict drug interactions. However, most of the methods cannot predict herb-drug interactions effectively.

Methods: Canonical SMILES of bioactive compounds was gathered from the PubChem online database, and its inhibition details were gathered PKCSM from the webserver.

Results: By searching the bioactive compound name in the search bar of "The HerbCYP450 Enzyme Inhibition Predictor online database" (HCIP- http://hcip.in/), it will provide the liver enzyme inhibition profile of the selected bioactive compound. For example; Guggulsterone: CYP3A4 inhibitor.

Conclusion: The Herb-CYP450 Enzyme Inhibition Predictor online database is very peculiar and easy to determine the inhibition profile of the targeted bioactive compound.

Keywords: CYP450; Enzyme inhibition; Bioactive Compounds; Online database; Herb-Drug Interaction

\section{Introduction}

Complementary and alternative medicine (CAM) practice usage has been increased throughout the world for various chronic illnesses. Plant-based medicinal products play a significant role in patient quality of life. The patient believes alternative therapies over their existing conventional treatment pattern since herbs are inherently safe, and they are self-prescribed. Majority of the patients having a habit of concomitant administration of alternative therapies with their existing conventional drugs without knowledge of their treating physician. ${ }^{1}$ The drug undergoes pharmacodynamics and pharmacokinetic reactions. Pharmacokinetics of a drug is essential for understanding the role of a drug in the human body. In the Pharmacokinetic parameters, the metabolism of the drug is crucial in framing the drug dosage and determining the action of the drug. The cytochrome CYP 450 superfamily plays a vital role in the metabolism of almost all drugs. There are 57 cytochromes identified in humans. Out of these six cytochromes, namely CYP1A2, CYP2B6, CYP2C9, CYP2C19, CYP2D6, and CYP3A4, metabolise $65 \%$ of the drug in the market. Induction or Inhibition of the
Cytochromes is the major cause of Drug Interactions. Herbal medicine will contain a number of active ingredients. ${ }^{2,3}$ The co-administration of herbal products and a drug can lead to severe alteration in the metabolism of the drug. The detection of this herb-drug interaction is expensive and higher time-consuming. Several computeraided techniques have been proposed in recent years to predict drug interactions. However, most of the methods cannot predict the herb-drug interactions effectively. Therefore knowing the exact cytochrome and its property as an inducer or inhibitor is crucial for drug research. The induction of the drug can lead to the early metabolism of the drug and further diminish the effect of a drug, where else the inhibitor of a specific enzyme will increase the activity of the drug, which can be either positive or negative on the clinical side.

\section{Overview of Herb-Drug Interaction:}

A single plant-based medicinal plant contains numerous bioactive compounds. There are higher chances of possible herb-drug interaction when plant-based medicinal products are administered together with conventional drugs. [Figure 1] 


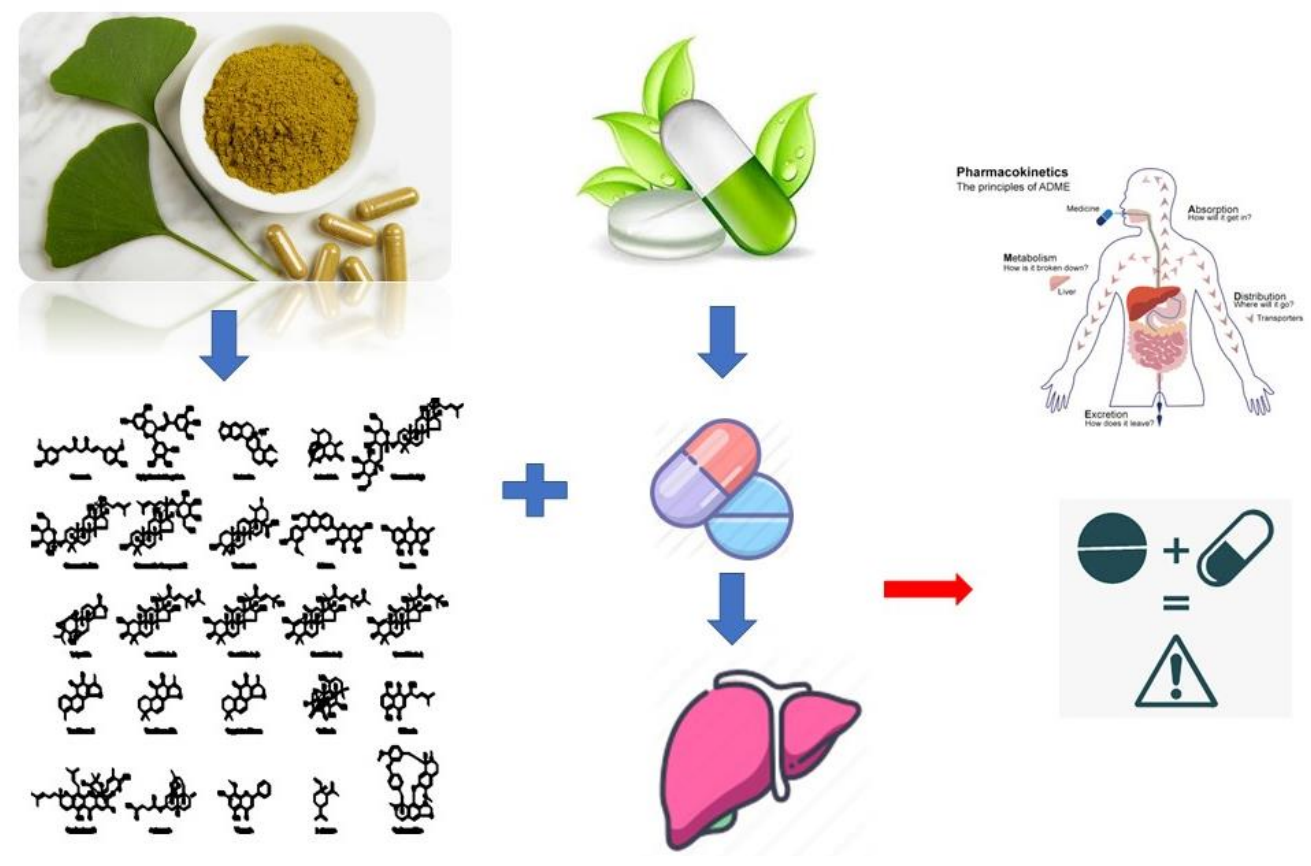

Figure 1: an overview of herb-drug interaction

\section{Materials and Methods:}

\section{HCIP Platform}

The sources for knowing the exact property of cytochrome enzymes in the metabolism of herbal medicines is still a tedious job. The accuracy of the obtained data and its reference is still in a dilemma. In order to resolve this, the Herb-CYP450 Enzyme Inhibition Predictor online database will make tracking very easy to identify the cytochrome enzyme and its property of bioactive compounds. The steps involved in this are very self-explaining. To build the database, we concentrate more on the CYP3A4 substrates as it metabolises $65 \%$ of the drug. Canonical SMILES of bioactive compounds was gathered from PubChem online database ${ }^{4}$, and its inhibition details were gathered PKCSM from the web server 5 . In order to avoid duplication, the extracted data were pre-processed. The processed data were classified as Cytochrome inhibitor and inducer. As per the PubChem database, If the AC50 value is less than or equal to $10 \mu \mathrm{M}$, then that compound is classified as a CYP inhibitor. Similarly, if the AC50 value is $>57 \mu \mathrm{M}$, the compound will be classified as CYP Inducer.

\section{Input \& Output Information}

The user has to enter the name of the compound in the search bar of the database. Optionally a user can select up to 1000 bioactive compounds for the prediction. The result will be generated instantly, and it will show the molecular weight and inhibition and substrate profile of the selected bioactive compound. [Figure 2]

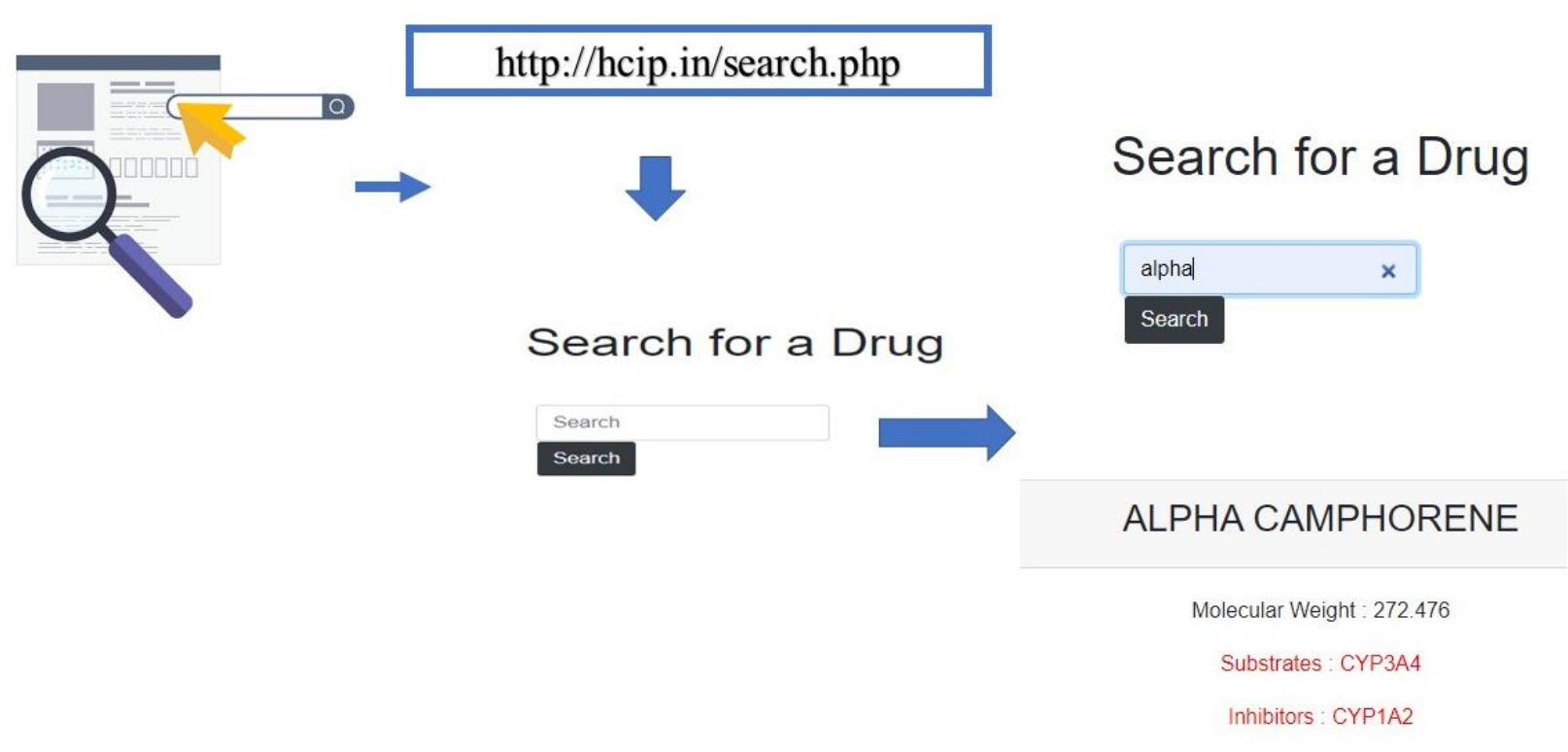

Figure 2: HCIP online database homepage 


\section{Discussion and Conclusion}

In the contemporary state, there has been a rapid rise in the use of herbal medicines along with conventional medicines. Estimation of herb-drug interaction is still challenging. Herb-drug interaction can be predicted based on the in vivo and in vitro methods. However, the existing in vivo technique is more time consuming and in vitro techniques are very expensive. Hence the new approach was made to evaluate the interaction potential of bioactive compounds with CYP3A4 enzyme using the online tool. The Herb-CYP450 Enzyme Inhibition Predictor online database will generate accurate information about the cytochrome activity of a selected bioactive compound in a very simple, user-friendly interference. The database was constructed based on trusted sources like PubChem, so the reliability of the information is at the core.

Declaration of Interest: The authors declare no conflict of interest in the contents of the manuscript.

Consent for Publication: Not applicable.

\section{Funding: None}

Conflict Of Interest: The authors declare no conflict of interest, financial or otherwise.

\section{Acknowledgements}

We would like to thank Shreejith S \& Sabarish Abishek W R for technical support.

\section{References}

1. Sarvesh, S., Koushik Muthu Raja, M., Rajanandh, M. G., \& Seenivasan, P. (2018). Prevalence and pattern of usage of complementary and alternative medicine among south Indian asthma patients in a tertiary care hospital. Complementary Therapies in Clinical Practice, 2018; 30:103108. doi:10.1016/j.ctcp.2017.12.016

2. Na, D. H., Ji, H. Y., Park, E. J., Kim, M. S., Liu, K.-H., \& Lee, H. S. Evaluation of metabolism-mediated herb-drug interactions. Archives of Pharmacal Research, 2011; 34:1829-1842. DOI: 10.1007/s12272-011-1105-0.

3. Steven X. Hu*, Chase A. Mazur and Kenneth L. Feenstra, "Assessment of Inhibition of Bovine Hepatic Cytochrome P450 by 43 Commercial Bovine Medicines Using a Combination of $<\mathrm{i}>$ In Vitro $</ \mathrm{i}>$ Assays and Pharmacokinetic Data from the Literature", Drug Metabolism Letters, 2019; 13:123. https://doi.org/10.2174/1872312813666191120094649

4. Kim, S., Chen, J., Cheng, T., Gindulyte, A., He, J., He, S., Li, Q., Shoemaker, B. A., Thiessen, P. A., Yu, B., Zaslavsky, L., Zhang, I., \& Bolton, E. E. (2019). PubChem in 2021: new data content and improved web interfaces. Nucleic acids research, 2021; 49(D1):D1388-D1395. https://doi.org/10.1093/nar/gkaa971

5. Douglas E. V. Pires, Tom L. Blundell, and David B. Ascher pkCSM: Predicting Small-Molecule Pharmacokinetic and Toxicity Properties Using Graph-Based Signatures Journal of Medicinal Chemistry 2015; 58 (9):4066-4072 